\title{
Rifampicin prophylaxis for throat carriage of Haemophilus influenzae type $b$ in patients with invasive disease and their contacts
}

\author{
Gwendolyn L Gilbert, Shona J MacInnes, Isobel A Guise
}

\begin{abstract}
Objective-To determine rates of colonisation with Haemophilus influenzae type b among household contacts of children with invasive $H$ influenzae type $b$ disease; compliance among medical staff with recommendations for use of rifampicin prophylaxis; and effects of rifampicin treatment in $H$ influenzae type b colonisation of patients and contacts.

Design-Prospective study of patients and their household contacts.

Setting-Royal Children's Hospital (the major paediatric hospital) in Victoria, Australia, with catchment population of 4.2 million, including 300000 children aged under 5 years.

Subjects-234 patients (age range 6 weeks to 8 years) with 235 episodes of all types of invasive $H$ influenzae type b disease admitted during 1988-9 and their contacts.
\end{abstract}

Main outcome measures-Positive cultures of $H$ influenzae type b from throat swabs taken at admission and six weeks subsequently; recording of rifampicin prophylaxis.

Results - The percentage of patients with positive throat cultures fell from $69 \%$ (33/48) on day 1 of admission to $9 \%(4 / 47)$ after three days' treatment; at six weeks' follow up $32 \%$ (32/99) of patients who had not received rifampicin and $8 \%(5 / 61)$ who had, had positive throat cultures. Among household contacts, $33 \%(62 / 190)$ of children and $7 \%(25 / 359)$ of adults were colonised, and the colonisation rates were similar in contacts of patients with all types of $\boldsymbol{H}$ influenzae type b disease. Rifampicin prophylaxis was indicated in 85 families; in $34 \%$ it was not prescribed at all for contacts and in $41 \%$ not as recommended. The colonisation rates at follow up were significantly less in siblings given rifampicin $(12 \%, 9 / 78)$, particularly in families in which all members were treated $(3 \%)$, than in those in which they were not $(36 \%)$ (odds ratio $21 \cdot 5 ; 95 \%$ confidence interval 3.0 to $103 \cdot 4$ ).

Conclusions - The rate of throat colonisation with $H$ influenzae type b was similar among siblings of children with all types of invasive $H$ influenzae type $b$ disease. Colonisation in contacts can be reduced by rifampicin prophylaxis, but some contacts remained colonised or were recolonised by the time of follow up. Medical staff complied poorly with current recommendations for rifampicin prophylaxis, which reduces its intrinsically limited value in preventing $H$ influenzae type b disease.

\section{Introduction}

Although epidemics of invasive Haemophilus influenzae type $b$ disease are rare, the incidence of the disease among susceptible close contacts of affected children is 500-600 times higher than in other children in the same age group..$^{1-4}$ Most secondary cases occur in the week after onset of disease in the index case; the risk is about $2 \%$ in contacts aged under 4 years and up to $5 \%$ in those aged under 2 years. ${ }^{13}$ If given appropriately, rifampicin prophylaxis may eliminate throat carriage of $H$ influenzae type $b$ and reduce the risk of secondary disease in contacts. ${ }^{5-7}$ On the basis of these observations the American Academy of Pediatrics and the National Health and Medical Research Council (Australia), among other authorities, recommend that if the contact group (usually a family) includes a child aged under 4 years, the whole group receive rifampicin prophylaxis (National Health and Medical Research Council, report of the 100th session, 1985). ${ }^{\times}$Despite the significantly increased risk of $H$ influenzae type $\mathrm{b}$ disease among close contacts, recognised secondary cases account for only $1-2 \%$ of cases, and rifampicin prophylaxis as recommended is time consuming; costly; and is associated with risk of side effects, possible development of rifampicin resistance by the organism, and, sometimes, failure to prevent disease. $^{910}$

Most contacts in whom $H$ influenzae type b colonisation and secondary disease have been studied were of patients with meningitis. Among the relatively few contacts of patients with epiglottitis studied, the trend was towards lower rates of colonisation than among those of patients with meningitis, ${ }^{112}$ However, the recommendations for prophylaxis do not distinguish between contacts of patients with different types of $H$ influenzae type $\mathrm{b}$ disease. Primary treatment of the invasive disease usually does not eliminate pharyngeal carriage of the organism by patients; after their discharge from hospital they are a potential source of bacteria for recolonisation of family contacts, unless they are also given rifampicin. ${ }^{13} 14$

We performed a study to determine: $(a)$ the rates of colonisation with $H$ influenzae type $b$ among household contacts of children with different types of invasive $H$ influenzae type b disease in Victoria, Australia, where the proportion of cases of epiglottitis is high ${ }^{15}$; (b) the extent to which current recommendations for rifampicin prophylaxis are followed by medical staff in a large children's hospital; $(c)$ the effects of treatment on $H$ influenzae type b colonisation of patients and of rifampicin prophylaxis on colonisation in both patients and contacts.

\section{Methods}

This study was part of a prospective study of invasive $H$ influenzae type $\mathrm{b}$ disease among children admitted to this hospital during 1988-9. Cases of invasive $H$ influenzae type b disease were defined as previously described, ${ }^{15}$ by isolation of $H$ influenzae type $b$ from a normally sterile specimen or from the upper respiratory tract of patients with epiglottitis or by detection of $H$ influenzae type $\mathrm{b}$ antigen in the urine or cerebrospinal fluid of a patient with clinically compatible disease.

As soon as possible after admission one or both parents were interviewed and asked to answer a questionnaire, which included detailed medical, family, and social histories (to be reported elsewhere). Throat swabs were taken from the patient and house-
Westmead Hospital,

Wales 2145, Australia.

BMF 1991;302:1432-5 
hold contacts while the patient was in hospital (if possible before rifampicin was given). If necessary, a research nurse visited the patient's home to collect the specimens. Follow up swabs were collected from the patient and household contacts during a visit to the family's home by the research nurse, about six weeks after the admission (usually at least four weeks after discharge).

Throat swabs were taken with a standard technique. The swab was placed into $1 \mathrm{ml}$ of Levinthal broth and used immediately to inoculate solid medium or stored at $4^{\circ} \mathrm{C}$ for no more than 24 hours. After inoculation the broth was shaken vigorously to release secretions, and the swab was removed and used to inoculate in a circular fashion around the edge of an agar medium containing $H$ influenzae type $\mathrm{b}$ antiserum and then discarded. With a sterile loop $1 \mu \mathrm{l}$ of the remaining broth was inoculated on to the agar in streaks radiating from its centre. ${ }^{16}$ The medium was Levinthal agar containing bacitracin $(0.25 \mathrm{~g} / \mathrm{l})$ and $5 \%$ hyperimmune burro antiserum (kindly provided by $\operatorname{Dr} M$ Glode, Children's Hospital, Denver, Colorado). The plates were examined after $24-36$ hours' incubation at $35^{\circ} \mathrm{C}$ in $5 \% \mathrm{CO}_{2}$ and suspect colonies were subcultured and identified by their growth requirements for $\mathrm{X}$ and $\mathrm{V}$ factors and by slide agglutination with commercial $H$ influenzae type $\mathrm{b}$ antiserum (Wellcome). Throat swabs were collected by house staff from some patients, mainly those with epiglottitis, in whom the swabs were taken at the time of intubation. These swabs were used to inoculate chocolate agar containing bacitracin. Suspect colonies were subcultured and identified as above. Isolates of $H$ influenzae type b were tested for $\beta$ lactamase production with a commercial nitrocefin disc (BBL) and for susceptibility to ampicillin, chloramphenicol, ceftriaxone, and rifampicin by a disc diffusion method; minimum inhibitory concentrations of these antibiotics for 120 isolates were subsequently determined by agar dilution.

Clinical staff caring for patients with invasive $H$ influenzae type disease had access to recommendations for use of rifampicin through locally published reviews and newsletters, discussion in hospital clinical meetings, or by consultation with the infectious disease team or pharmacy department. ${ }^{1718}$ We did not attempt to influence, directly, the prescribing of rifampicin to patients or their contacts, or both, during the study. Whether rifampicin was prescribed or not was determined from the patient's record and whether it had been taken by questioning the parents. The recommended dose was $20 \mathrm{mg} / \mathrm{kg}$ daily for four days.

Comparison of proportions were made using the $\chi^{2}$ test or, when numbers were small, Fisher's exact test. Odds ratios and $95 \%$ confidence intervals were calculated with the Epistat statistical package.

\section{Results}

During the study period 234 patients were admitted with 235 episodes of $H$ influenzae type b disease. Their ages ranged from 6 weeks to 8 years; $120(51 \%)$ were aged under 2 years and $40(17 \%)$ were aged over 4 years. The clinical manifestations were: meningitis $(83$ patients, one of whom had two episodes at ages 6 and 8 months; mean age 22.6 months); epiglottitis ( 94 ; mean age $37 \cdot 1$ months); pneumonia (18); cellulitis (17); bacteraemia (13); septic arthritis (6); and pericarditis, severe pharyngitis, and a thyroglossal cyst (one each). The mean age of patients with infections other than meningitis or epiglottitis was 26.6 months. Two patients with meningitis and one with epiglottitis died. Most patients $(80 \%)$ were treated with chloramphenicol, including $73(88 \%)$ of those with meningitis and 93 $(99 \%)$ of those with epiglottitis (the only exception was a patient who died suddenly before receiving anti- biotics). Eight patients with meningitis were treated with ceftriaxone and two with cefotaxime. Among patients with other infections, $21(37 \%)$ were treated with chloramphenicol and $30(53 \%)$ with amoxycillin (two patients had both); 41 patients had combinations of two different antibiotics and 16 patients three. The clinical details of these patients will be reported elsewhere.

All 120 isolates of $H$ influenzae type $\mathrm{b}$ were susceptible to chloramphenicol, ceftriaxone, and rifampicin by disc diffusion testing; 33 (14\%) isolates produced 6 lactamase and an additional two were resistant to ampicillin by disc diffusion testing but did not produce $b$ lactamase.

Patients' throat swabs collected on admissionThroat swabs from 193 (82\%) patients were cultured. $H$ influenzae type b was isolated from the swabs of $10 \%$ (7/68) of patients with meningitis, $41 \%(33 / 80)$ of patients with epiglottitis, and $31 \%(14 / 45)$ of patients with other types of infection. The low isolation rate from patients with meningitis correlated with delay in collecting the specimens. The isolation rate was $69 \%$ when swabs were collected on the day of admission but only $9 \%$ for swabs taken after three days of parental antibiotic treatment (table I). Specimens were not collected from some patients, particularly those with infections other than meningitis and epiglottitis, until more than a week after admission.

A total of $63(27 \%)$ of the 235 patients had had some oral antibiotic treatment before admission, including 32 of $84(38 \%)$ with meningitis, 11 of $94(12 \%)$ with epiglottitis, and 20 of 57 (35\%) patients with other infections. Antibiotic treatment before admission did not appreciably affect the rate of isolation of $H$ influenzae type $b$ from the throat swab. In patients whose throat were swabbed positive cultures resulted in 14 of 51 $(27 \%)$ of those who had had antibiotics and 40 of 142 (28\%) who had not.

Initial throat swabs from contacts - Throat swabs were collected during the patient's admission from 166 mothers, 148 fathers, and 45 other adult household contacts; 15 (9\%) mothers, six (4\%) fathers, and four $(9 \%)$ other adults ( $7 \%$ of all adult contacts) had positive cultures. One hundred and seventy eight $(76 \%)$ of the patients had at least one sibling. Two children in one family had $H$ influenzae type $\mathrm{b}$ disease: both had epiglottitis and presented within 24 hours of each other - that is, they were coprimary cases. Altogether, there were 292 siblings and 234 index patients in 233 families (mean 2.3 children per family, including the index patient); $92(32 \%)$ of the siblings were aged under 4 years. The number and age distribution of siblings did not differ significantly between contacts of patients with meningitis, epiglottitis, and other types of infection (table II). Initial throat swabs were collected from $190(65 \%)$ siblings and $13(33 \%)$ were positive. The proportion of contacts with positive throat swabs did not differ significantly according to the type of disease in the index patient (table II).

Rifampicin "prophylaxis"-Table III summarises the use of rifampicin in household contacts of patients. Rifampicin was prescribed for some or all members of

TABLE I-Throat carriage of $H$ influenzae type $b$ in patients with invasive disease

\begin{tabular}{lcccc}
\hline \multirow{2}{*}{$\begin{array}{l}\text { Hospital } \\
\text { day }\end{array}$} & \multicolumn{4}{c}{ Positive throat swabs/total cultured } \\
\cline { 2 - 5 } & Meningitis & Epiglottitis & Other infections & Total (\%) \\
\hline 1 & $3 / 7$ & $29 / 38$ & $1 / 3$ & $33 / 48(69)$ \\
2 & $2 / 10$ & $2 / 17$ & $1 / 4$ & $5 / 31(16)$ \\
3 & $0 / 19$ & $1 / 10$ & $3 / 9$ & $4 / 38(11)$ \\
$4-7$ & $1 / 22$ & $1 / 9$ & $2 / 16$ & $4 / 47(9)$ \\
$>7$ & $1 / 9$ & $0 / 6$ & $6 / 12$ & $7 / 27(26)$ \\
Unknown & $0 / 1$ & - & $1 / 1$ & $1 / 2$ \\
\hline Total (\%) & $7 / 68(10)$ & $33 / 80(41)$ & $14 / 45(31)$ & $54 / 193$ \\
\hline
\end{tabular}


TABLE II - Throat carriage of $H$ influenzae type $b$ in siblings of patients with invasioe disease

\begin{tabular}{|c|c|c|c|c|c|}
\hline Disease & $\begin{array}{l}\text { No of } \\
\text { cases }\end{array}$ & $\begin{array}{l}\text { No } \%) \\
\text { with } \\
\text { siblings }\end{array}$ & $\begin{array}{l}\text { Total } \\
\text { No of } \\
\text { siblings }\end{array}$ & $\begin{array}{l}\text { No } \% \\
\text { siblings aged } \\
<+ \text { years }\end{array}$ & $\begin{array}{c}\text { Positive throat } \\
\text { swabs/Not taken } \\
(\%)\end{array}$ \\
\hline Meningiti & 84 & 63,75 & 98 & $34(35)$ & $23 / 75 \quad 31)$ \\
\hline Epiglottitis & 94 & $70 \div 74$ & 121 & $33(27)$ & $21 / 69 \quad 30$ \\
\hline Other infections & 57 & 45,80 & 73 & $25(34)$ & $18 / 46 \quad 39$ \\
\hline Total & 235 & $178(76)$ & 292 & $92(32)$ & $62 / 190(33)$ \\
\hline
\end{tabular}

TABLE III-Use of rifampicin in families and contacts of patients with invasive $H$ influenzae type b disease

\begin{tabular}{lr}
\hline & $\begin{array}{r}\text { No }(\%) \\
\text { of families }\end{array}$ \\
\hline $\begin{array}{l}\text { Other children in family aged }<4 \text { years }(\mathrm{n}=85): \\
\text { Receiving rifampicin }\end{array}$ & \\
$\quad$ None & $24(28)$ \\
$\quad$ Patients only & $5(6)$ \\
$\quad$ Some or all contacts & $56(66)$ \\
No other children in family aged $<4$ years $(\mathrm{n}=149):$ & \\
Receiving rifampicin & $106(71)$ \\
$\quad$ None & $25(17)$ \\
$\quad$ Patients only & $18(12)$ \\
$\quad$ Some or all contacts & \\
Some or all contacts receiving rifampicin $(\mathrm{n}=74):$ & $44(59)$ \\
All contacts and patient & $9(12)$ \\
Siblings only & $8(11)$ \\
Siblings and parents & $13(18)$ \\
Siblings and patient & \\
\hline
\end{tabular}

$66 \%$ of families in which there was a child, other than the index patient, under 4 years of age and in $12 \%$ of families in which there was not. In families for which it was prescribed the use of rifampicin was variable; it was prescribed for all members, including the index patient, in only $59 \%$. Rifampicin was not prescribed for contacts in $34 \%(29 / 85)$ of families in whom it was indicated according to current recommendations; these included $25 \%(8 / 32)$ of families of patients with meningitis, $20 \%(6 / 30)$ of those with epiglottitis, and $65 \%(15 / 23)$ of those with other infections. The proportion of families of patients with infections other than meningitis and epiglottitis who were not given rifampicin (when it was indicated) was significantly higher than those of patients with either meningitis or epiglottitis $(p=0 \cdot 006$; odds ratio $6 \cdot 4,95 \%$ confidence interval $2 \cdot 0$ to $21 \cdot 1$ ). Among 74 families in which rifampicin was prescribed for some or all members, 18 had no child aged under 4 years other than the index patient including $36 \%(14 / 38)$ of families of patients with meningitis, $11 \%(3 / 27)$ of those with epiglottitis, and $11 \%(1 / 9)$ of those with other infections.

Effect of rifampicin on throat carriage in patients and contacts-Positive throat swabs resulted from 34\% (34/99) of swabs from patients who had not been given rifampicin and $8 \%(5 / 61)$ from those who had been given rifampicin $(p=0.0005 ; 5.8,2.0$ to $18 \cdot 1)$. In families in which no rifampicin was given or it was given only to the index patient the proportion of positive throat swabs from contacts did not differ between initial and follow up cultures $(8 \%(16 / 209)$ initial and 7\% (11/200) follow up swabs in adults and $40 \%(44 / 109)$ and $36 \%(39 / 108)$ respectively in children). Isolation of $H$ influenzae type $b$ from follow up swabs in adults, in those families in which rifampicin prophylaxis was given, was reduced from $7 \%$ to $2 \%$. The initial colonisation rate was significantly lower among siblings given rifampicin than among those not $(22 \%, 18 / 81 \vee 40 \%, 44 / 109 ; \mathrm{p}=0.01$; odds ratio $2 \cdot 4$, $95 \%$ confidence interval $1 \cdot 2$ to $4 \cdot 8)$. This reflects the fact that in two thirds of families swabs were collected after at least one dose of rifampicin had been taken The colonisation rate at follow up $(12 \%, 9 / 78)$ was not significantly different from the initial rate in these patients $(p=0.06)$ but was significantly lower than the follow up colonisation rate in siblings not given rifampicin $(36 \%, 39 / 108 ; \mathrm{p}<0 \cdot 003$; odds ratio $4 \cdot 3,95 \%$ confidence interval 1.8 to 10.5 ). In the families in which all members received rifampicin only $3 \%(1 / 39)$ of children were colonised at follow up; among siblings who were given rifampicin but either whose parents or the index patient were not $21 \%(8 / 39)$ were colonised at follow up, which was not quite significantly different from that in children not treated with rifampicin $(36 \%$, 39/108; $p=0 \cdot 053$ ).

\section{Discussion}

We assumed that pharyngeal colonisation with $H$ influenzae type $\mathrm{b}$ precedes invasive disease. The isolation rate of only $70 \%$ from patients whose throat swabs were taken on the day of admission probably reflects the fact that a single swab is relatively insensitive for detecting colonisation, especially if cultured on chocolate agar containing bacitracin rather than antiserum agar, ${ }^{16}{ }^{19}$ and that many patients had been given at least one dose of intravenous antibiotic treatment (usually chloramphenicol) before the swab was taken. The rapid decrease in the proportion of patients with swabs giving positive results reflects the ability of chloramphenicol, particularly, to suppress throat colonisation $^{20}$; this presumably reduces the risk of nosocomial transmission of $H$ influenzae type $\mathrm{b}$ to a negligible level after the first day of treatment. The fact that most patients with disease other than meningitis or epiglottitis were treated with amoxycillin (which is less active than chloramphenicol in suppressing colonisation with $H$ influenzae type b) probably explains the relatively high colonisation rate after several days of treatment..$^{20}$

Colonisation rates of $40 \%$ in child contacts not given rifampicin and $7 \%$ in adult contacts were lower than those reported in some other studies. ${ }^{7112}$ In one study $71 \%$ of patients aged under 4 years and $44 \%$ of $5-10$ year olds were colonised." The colonisation rate varies with household crowding. ${ }^{12}$ Some reports suggested that carrier rates are lower in contacts of patients with epiglottitis than in those of patients with meningitis (39\% v 56\% in one study and $14 \%$ v $27 \%$ in another), although the numbers of patients with epiglottitis were too small to show significance. ${ }^{112}$ In this study colonisation rates in the contacts of patients with epiglottitis did not differ significantly from those of patients with meningitis or other infections.

The use of rifampicin in contacts varied considerably. It was not clear whether this was owing to misinterpretation of the guidelines or use of alternative protocols. Rifampicin was prescribed least frequently, when indicated, in contacts of patients with infection other than meningitis and epiglottitis, presumably because the bacterial aetiology was not immediately obvious. It was more likely to be given when not indicated in contacts of patients with meningitis, which may reflect greater anxiety associated with this disease. The results confirm the ability of rifampicin, if given as recommended, to eradicate throat colonisation by $H$ influenzae type $\mathrm{b}$ in most of those treated. ${ }^{5-721}$ However, the fact that the colonisation rate was relatively high at follow up among siblings who were given rifampicin but exposed to untreated household contacts emphasises the risk of colonisation. Previous studies showed that the use of rifampicin can protect contacts of patients with invasive $H$ influenzae type b disease but, because of the fairly small proportion of cases which are attributable to secondary spread, its use has little impact on the overall incidence of the disease. $^{6}$ There were no secondary cases (and only one coprimary case) among the patients in this study. In a previous study of 547 cases of invasive $H$ influenzae type $b$ disease in Victoria, three pairs of siblings, all aged under 5 years, were affected. Rifampicin pro- 
phylaxis had not been given to contacts of these patients, but only one of the "secondary" cases occurred within 30 days of the index case; the others were two months and three months apart. Although rifampicin prophylaxis is of limited value, the relatively poor compliance with the current recommendations suggests that its value is commonly reduced even further by incorrect use.

There has been concern that widespread use of rifampicin might lead to the development of rifampicin resistant strains of $H$ influenzae type $b .^{10}$ However, in our laboratory all of 120 strains of $H$ influenzae type b tested by agar dilution were inhibited by $<0: 03 \mathrm{mg} / \mathrm{l}$ rifampicin (unpublished data). Other objections to the use of rifampicin are that it sometimes fails to prevent secondary $H$ influenzae type $\mathrm{b}$ disease ${ }^{9}$ and that it is associated with significant cost and risk of toxicity. A four day course for a family of two adults and three children is \$A50 and the risk of toxicity is small if suitable precautions are observed. At present there is no alternative to rifampicin for contacts in whom its use is contraindicated - for example, pregnant women. Ceftriaxone is effective in eliminating Neisseria meningitidis from the throats of carriers. ${ }^{22}$ With the more widespread use of this agent for treating invasive $H$ influenzae type $\mathrm{b}$ disease it would be useful to determine whether it can also eliminate this organism from the throats of patients; if so, it may be an alternative to rifampicin for selected contacts.

This study has emphasised the limitations of antibiotic prophylaxis in preventing $H$ influenzae type $\mathrm{b}$ disease. A significant impact on its incidence can be achieved only by immunisation,,$^{23}$ which should be introduced as soon as possible for protection of children at risk.

1 Filice GA, Andrews JS, Hudgins MP, Fraser DW. Spread of Haemophilus influenzae. Secondary illness in household contacts of patients with $\mathrm{H}$ influenzae meningitis. Am $\mathcal{F}$ Dis Child 1978;132:578-9.

2 Ward JI, Fraser DW, Baraff LJ, Plikaytis BD. Haemophilus influenzae meningitis. A national study of secondary spread in household contacts. N Engl F Med 1979;301:122-6.
3 Glode MP, Daum RS, Goldmann DA, Leclair J, Smith A. Haemophilus influenzae type $b$ meningitis: a contagious disease of children. $B M J$ 1990;280:899-901.

4 Campbell LR, Zedd AJ, Michaels RH. Household spread of infection due to Haemophilus influenzae type b. Pediatrics 1980;66:115-7.

5 Glode MP, Daum RS, Boies EG, Ballard TL, Murray M, Granoff DM. Effect of rifampicin chemoprophylaxis on carriage eradication and new acquisition of Haemophilus influenzae type b contacts. Pediatrics 1985;76:537-42.

6 Band JD, Fraser DW, Ajello G. Prevention of Haemophilus influenzac type b disease. FAMA 1984;251:2381-6.

7 Centers for Disease Control. Prevention of secondary cases of Haemophilus influenzac type b disease. MMWR 1982;31:672-4, 679-80.

8 American Academy of Pediatrics, Committee on Infectious Diseases. Revision of recommendation for use of rifampicin prophylaxis of contacts of patients of recommendation for use of rifampicin prophylaxis of contacts of
with Haemophilus influenzae infection. Pediatrics 1984;74:301-2.

9 Bodies EG, Granoff DM, Squires JE, Barenkamp SJ. Development of Haemophilus influenzae type $b$ meningitis in a household contact treated with rifampicin. Pediatrics 1982;70:141-2.

10 Murphy TV, McCracken GH, Zweighaft TC, Hansen EJ. Emergence of rifampicin-resistant Haemophilus influenzae after prophylaxis. I Pediat 1981;99:406-9.

11 Michaels RH, Norden CW. Pharyngeal colonization with Haemophilus influenzae type b: a longitudinal study of families with a child with meningitis or epiglottitis due to $\mathrm{H}$ influenzae type $\mathrm{b}$. 7 Infect $D_{\text {ts }}$ 1977;136:2228.

12 Daum RS, Glode MP, Goldmann DA, et al. Rifampicin chemoprophylaxis fo household contacts of patients with invasive infections due to Haemophilus influenzae type b. F Pediatr 1981;98:485-91.

13 Gilsdorf JR. Dynamics of nasopharyngeal colonization with Haemophilus influenzae type b during antibiotic therapy. Pediatrics 1986;77:242-5.

14 Apert GA, Campos JM, Smith DR, Barenkamp SJ, Fleisher GR. Incidence and persistence of Haemophilus influenzae type b upper airway colonization and persistence of Haemophilus influenzae type b upper
in patients with meningitis. F Pediatr 1985;107:555-7.

15 Gilbert GL, Clements DA, Broughton AJ. Haemophilus influenzae type b infections in Victoria, Australia, 1985-1987. Pediatr Infect Dis $\mathcal{F}$ 1990;9:252-7.

16 Michaels RH, Stonebraker FE, Robbins JB. Use of antiserum agar for detection of Haemophilus influenzae type $\mathrm{b}$ in the pharynx. Pediatr Res 1975;9:513-6.

17 Gilbert GL. Epidemiology and prevention of invasive Haemophilus influenzac type b infection. Aust Paediatr f 1987;23:323-7.

18 Gilbert GL. Antibiotic prophylaxis for contacts of patients with invasive infection due to Haemophilus influenzae type b. Australian College of Paediatrics Newsletter, May, 1987.

19 Michaels RH, Poziviak CS, Stonebraker FE, Norden CW. Factors affecting pharyngeal Haemophilus influenzae type b colonization rates in children. Y Clin Microbiol 1976;4:413-7.

$20 \mathrm{Li} \mathrm{KI}$, Wald ER. Use of rifampicin in Haemophilus influenzae type $r$ infections. Am F Dis Child 1986;140:381-5.

21 Shapiro ED, Wald ER. Efficacy of rifampicin in eliminating pharyngeal carriage of Haemophilus influenzae type b. Pediatr 1980;66:5-8.

22 Schwartz B, Al-Tobaiqi A, Al-Ruwais. et al. Comparative efficacy of ceftriaxone and rifampicin in eradicating pharyngeal carriage of group A Neisseria meningitidis. Lancet 1988;i:1239-42.

23 Clements DA, Gilbert GL. Immunization for prevention of Haemophilus influenzae type b infection: a review. Aust NZ F Med 1990;20:828-34.

(Accepted 12 April 1991)

\section{Audit of an official recommendation on screening for congenital dislocation of the hip}

\author{
Dai Anthony Jones, Dawn Beynon, \\ B N C Littlepage
}

Guidance on screening for congenital dislocation of the hip has been given by the Standing Medical Advisory Committee for the Central Health Services Council, which reported in 1966 and 1969.' In 1986 this advice was updated. ${ }^{2}$ The booklet was made widely available and also stimulated editorials and special articles in leading medical journals. ${ }^{3+}$ This report was published against a background of doubt that screening was effective and also evidence that the incidence of late cases was increasing despite screening.

The 1986 recommendations recognised that several health professionals may take part in the screening process, and therefore recommended that " $A$ designated officer within each Health District should keep the whole Screening programme under review and record and evaluate the incidence of cases detected late - such information should be made available to the health professionals concerned." Although the term "audit" was not widely used at the time, it is clear that this is what was being proposed-that is, feedback of the failures of the process of screening and modification of the process accordingly. We assessed the effectiveness of the distribution of the recommendation and its level of implementation.

\section{Methods and results}

A questionnaire was sent to each health district in the United Kingdom. Out of a wide range of questions asked, two were: "Have you identified a designated officer within your district to keep the whole screening programme under review?" and "Does any paediatrician or orthopaedic surgeon in your district have a special interest in congenital dislocation of the hip? (Please name.)"

Of the 216 questionnaires distributed, 152 health districts replied (70\%). Only $67(45 \%)$ of these health districts had appointed a designated officer to oversee the screening process. Of the 150 replies, 95 named clinicians who had a special interest in congenital dislocation of the hip: 75 were orthopaedic surgeons, 13 were paediatricians, one was a community physician, and the others quoted a combination of specialties.

\section{Comment}

The revised memorandum on screening for congenital dislocation of the hip was widely circulated to professional groups and health authorities accompanied by a letter from the chief medical officer or chief nursing officer $(\mathrm{C} 190(86) 17, \mathrm{CNO}(86) 13)$. A press 